\title{
Coordinated Droop Control and Adaptive Model Predictive Control for Enhancing HVRT and Post-Event Recovery of Large-Scale Wind Farm
}

Wei, Juan; Cao, Yijia; Wu, Qiuwei; Li, Canbing; Huang, Sheng; Zhou, Bin; Xu, Da

Published in:

IEEE Transactions on Sustainable Energy

Link to article, DOI:

10.1109/TSTE.2021.3053955

Publication date:

2021

Document Version

Peer reviewed version

Link back to DTU Orbit

Citation (APA):

Wei, J., Cao, Y., Wu, Q., Li, C., Huang, S., Zhou, B., \& Xu, D. (2021). Coordinated Droop Control and Adaptive Model Predictive Control for Enhancing HVRT and Post-Event Recovery of Large-Scale Wind Farm. IEEE Transactions on Sustainable Energy, 12(3), 1549 - 1560. https://doi.org/10.1109/TSTE.2021.3053955

\section{General rights}

Copyright and moral rights for the publications made accessible in the public portal are retained by the authors and/or other copyright owners and it is a condition of accessing publications that users recognise and abide by the legal requirements associated with these rights.

- Users may download and print one copy of any publication from the public portal for the purpose of private study or research.

- You may not further distribute the material or use it for any profit-making activity or commercial gain

- You may freely distribute the URL identifying the publication in the public portal 


\title{
Coordinated Droop Control and Adaptive Model Predictive Control for Enhancing HVRT and Post-Event Recovery of Large-Scale Wind Farm
}

\author{
Juan Wei, Yijia Cao, Senior Member, IEEE, Qiuwei Wu, Senior Member, IEEE, Canbing Li, Senior \\ Member, IEEE, Sheng Huang, Bin Zhou, Senior Member, IEEE, Da Xu
}

\begin{abstract}
The wind turbine (WT) terminal overvoltage during grid voltage swell events may result in tripping the WT and consequently threaten the secure operation of large-scale wind farms (WFs). In this paper, an optimal coordination of droop control and adaptive model predictive control (MPC) scheme is proposed to enhance the high-voltage ride-through (HVRT) and post-event recovery of large-scale WFs. During the HVRT, the reactive power reference is generated in each WT controller by following an optimal droop coefficient to realize a fast voltage reduction at the $W T$ terminal. The droop coefficients are precalculated by taking the WF collection system topology and voltage swell magnitude into consideration. At the post-event recovery stage, an adaptive MPC-based voltage recovery control scheme is proposed to improve post-event voltage dynamic restoration performance. The droop coefficients of the WT controllers are optimized based on the voltage sensitivity coefficients and voltage swell magnitude. With the proposed control scheme, all the WT terminal voltage can be maintained within their feasible range and the response time of post-event voltage recovery is significantly shortened. The proposed control scheme is validated and tested under various operating scenarios.
\end{abstract}

Index Terms-Wind farm, high-voltage ride-through (HVRT), post-event recovery, model predictive control, droop control

\section{INTRODUCTION}

$\mathrm{W}$ ITH the increase of wind power penetration and the growing of the wind farms (WFs) size, the secure operation and auxiliary services of the WF have received widespread attention [1]-[2]. Since large-scale WFs are usually located far from load centers, the short circuit ratio (SCR) of the system is low, usually under 3 [3]-[4]. Thus, the weak grid disturbance may trigger the cascading trip of wind turbines (WTs). The voltages of WT terminals and point of connection (POC) are key indicators of power quality in large-scale WFs,

This work was supported by the Sino-US International Science and Technology Cooperation Project under grant no. 2019YFE0114700.

J. Wei, Y Cao, and B. Zhou are with the College of Electrical and Information Engineering and the Hunan Key Laboratory of Intelligent Information Analysis and Integrated Optimization for Energy Internet, Hunan University, Changsha 410082, China (e-mail: weijuanba@hnu.edu.cn; yjcao@ hnu.edu.cn; binzhou@hnu.edu.cn).

C. $\mathrm{Li}$ is with the School of Electronic Information and Electrical Engineering, Shanghai Jiaotong University, Shanghai 200240, China (e-mail: licanbing@sjtu.edu.cn).

Q. Wu and S. Huang are with the Center for Electric Power and Energy, Department of Electrical Engineering, Technical University of Denmark (DTU), Kongens Lyngby2800, Denmark (e-mail: qw@elektro.dtu.dk, huang98123@163.com ).

D. $\mathrm{Xu}$ is with the School of Automation, China University of Geosciences, Wuhan 430074, China, and also with Hubei Key Laboratory of Advanced Control and Intelligent Automation for Complex Systems, Wuhan 430074, China (e-mail: eedaxu@outlook.com). which may encounter serious fluctuations caused by inherent intermittency and volatility features of wind energy [5]-[6]. In particular, the WT terminal overvoltage during grid voltage swell events may result in the WT tripping and threaten the operation of large-scale WFs consequently.

In a WF, voltage swells may result from energizing submarine cables, switching off a large proportion of loads, charging capacitor banks, and asymmetric faults [7]-[9]. When far-end events occur in a weak grid, the voltages inside the WFs vary with the gird voltage swell event. Moreover, the fluctuations of voltages inside the WF caused by the variation of wind speed and low $\mathrm{X} / \mathrm{R}$ ratio of the medium voltage bus may lead to more serious consequences during the event [10]. In order to overcome these challenges, the impact of grid voltage swell on WTs and relevant high-voltage ride-through (HVRT) strategies should be investigated thoroughly.

One of the existing solutions to improve the HVRT capability is to add external auxiliary equipment. A vectorbased hysteresis current regulator has been used to mitigate the rotor current oscillations and improve the fault-ride-through performance of doubly-fed induction generator (DFIG)-based WTs [11]. In [12], a chopper protection circuit was used to consume excessive power and maintain DC-link voltage stable, which reduced the wind power system efficiency due to heat losses of the resistor. In [13], a virtual impedance control strategy was proposed to mitigate rotor current oscillations and improve the HVRT capability of the WT. In [14], the coordination of series dynamic resistor devices and crowbar protection was adopted to reduce rotor circuit voltage and limit the current of the DFIG-based large-scale WFs. In [15], a reverse-current tracking control strategy without flux linkage and system parameters was proposed to accelerate the decay rate of the transient flux during the HVRT. Previous control schemes mainly focus on their feasibility and effectiveness in current control and voltage stability issues for the single WT. However, in a practical WF, the terminal voltages of WTs are different due to their different locations inside the WF collection system. Besides, the terminal voltage of each WT also depends on reactive power injections of all WTs inside the WF. Especially when grid voltage swells occur, the terminal voltages of WTs may fluctuate heavily even exceeding their thresholds set by grid codes. Therefore, the coordination among WTs is necessary to achieve the optimal operation of the WF under grid voltage swell conditions. However, the interaction among different WTs and the influence among the WF and WTs during the HVRT and post-event voltage recovery period 
have not been considered.

Extensive studies on the voltage/Var control schemes of WFs have been studied in [16]-[20]. In [16], the reactive power requirement of the WFs was calculated according to the states at the point of common coupling (PCC) and dispatched to all WTs based on the proportional distribution of the maximum reactive power. In [17]-[18], a model predictive control (MPC)based centralized voltage control strategy was proposed to minimize voltage deviations for VSC-HVDC connected offshore WFs. The optimal coordination among WTs and various Var devices with different time scales were also investigated. In [19]-[20], the proposed distributed control scheme can effectively reduce control complexity and facilitate system management, which is applied and verified in practical projects. In [21], an optimization algorithm based on the alternating direction method of multipliers (ADMM) to solve the computational efficiency and fast convergence problem for WFs with a two-tier control structure was proposed. In [22], a distributed voltage control scheme based on the analytical target cascading (ATC) algorithm was proposed for a large-scale WF cluster. The sensitivity-based voltage optimization problem was decomposed and the exchanged information only included the shared control variables with adjacent sub-WF controllers. However, the dynamic voltage characteristics of the WFs under voltage swell events and the interactions between the WFs and WTs during the HVRT and post-event recovery period remains blank.

To address the overvoltage of the WT terminal during voltage swell events, an optimal coordination of droop control and adaptive model predictive control scheme is proposed in this paper for the HVRT and post-event recovery enhancement of large-scale WFs. The contributions of this paper are summarized as follows:

1) During the HVRT, an optimal droop control scheme is developed to regulate the reactive power output of WTs considering the variation of the wind speed and electrical distance among WTs. The control algorithm can identify unique optimal droop coefficients for individual WTs rather than using the same droop coefficients for all WTs, which can maintain all the WT terminal voltages within their feasible range specified by general grid code while fast reducing voltage deviations.

2) The droop coefficients during the HVRT are precalculated by taking the WF collection system topology and voltage swell magnitude into consideration to achieve a global optimal operational performance, while effectively reducing heavy communication and calculation burden.

3) In the post-event recovery process, an adaptive MPCbased voltage recovery control (AMVRC) scheme is designed to rapidly respond to the WT terminal voltage fluctuations and improve post-event voltage dynamic restoration performance. The MPC optimization algorithm is used to further optimize the reactive power outputs of WTs and minimize WT terminal voltage derivations. Compared with conventional centralized control, the control period of the WF can be extended, thereby potentially reducing the calculation burden of the WF central controller and impacts on communication delay.

The rest of this paper is organized as follows. The problem statement of HVRT and post-event recovery processes is presented in Section II. Section III gives the control architecture of the proposed scheme and the modeling of the WF. In Section IV, the HVRT and post-event recovery control schemes are described in detail. Comparative studies are shown and discussed in Section V, followed by the conclusions.

\section{Problem Statement of HVRT and Post-eVent RECOVERY PROCESSES}

Fig. 1 depicts the configuration of a WF, which consists of 32 type-4 WTs with $5 \mathrm{MW}$ rated capacity. Each WT is connected to the feeder through a $0.9 / 33 \mathrm{kV}$ transformer. There are four feeders connected to the medium voltage (MV) bus and eight WTs in each feeder placed every $4 \mathrm{~km}$. All of WTs are collected at the POC and delivered to external grids by the $155 / 380 \mathrm{kV}$ main transformer.

Under normal operation, the central controller of the WF generates the reactive power reference for WTs to regulate the bus voltages inside the WF or provide the reactive power support for the external grid. Since this paper concentrates on the HVRT and post-event voltage recovery of the WF, the control schemes under normal operation are not given in detail. Once a grid voltage swell event occurs, the terminal voltage of the WT will rapidly increase with the grid voltage increasing. The overvoltage at each WT terminal results in high mechanical and electrical stress for WTs and converters due to the transient natural flux [13], which endangers the secure operation of WF consequently. Thus, the WF should initiate a controlled voltage drop for the WT terminal overvoltage. After the event clearance, the WT terminal voltage should be recovered to the feasible range as soon as possible.

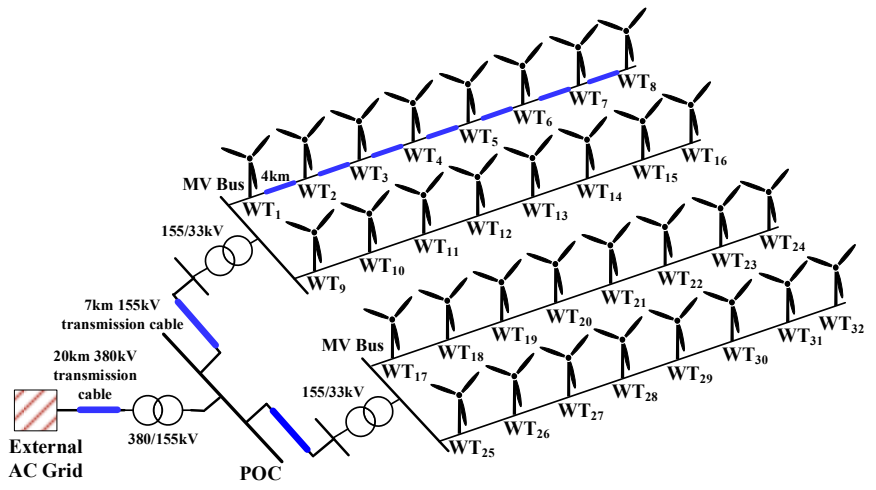

Fig. 1. WF Configuration

The latest Wind Turbines-Test Procedure of Voltage Fault Ride Through Capability includes the HVRT and LVRT requirements in China as shown in Fig. 2 [23]. The WTs are required to continuously operate in the area between the HVRT curve and the LVRT curve, otherwise the WTs may be tripped off. The reactive current injection requirement of the German E. ON Netz code, shown in Fig. 3 [24], is adopted. Defining the set of $n$ WTs as $N_{\mathrm{W}}=\{1,2, \cdots, j, \cdots, n\}$, the relationship between the voltage amplitude $V_{\mathrm{Wj}}$ and time duration $T_{\mathrm{Wj}}$ can be presented as,

$$
T_{\mathrm{W} j} \leq\left\{\begin{array}{ll}
0.5 \mathrm{~s} & V_{\mathrm{W}_{j}} \in(1.25,1.3] \text { p.u. } \\
1.0 \mathrm{~s} & V_{\mathrm{w}_{j}} \in(1.2,1.25] \text { p.u. } \\
10 \mathrm{~s} & V_{\mathrm{w}_{j}} \in(1.1,1.2] \text { p.u. }
\end{array} \quad \forall j \in N_{\mathrm{w}}\right.
$$




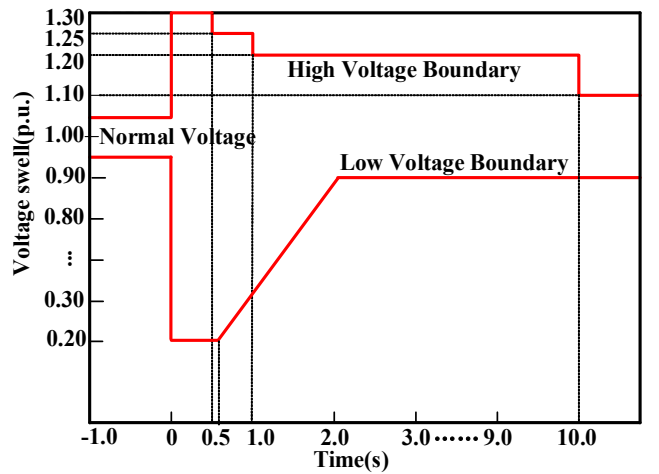

Fig. 2. Grid code requirement for WT HVRT and LVRT capability in China.

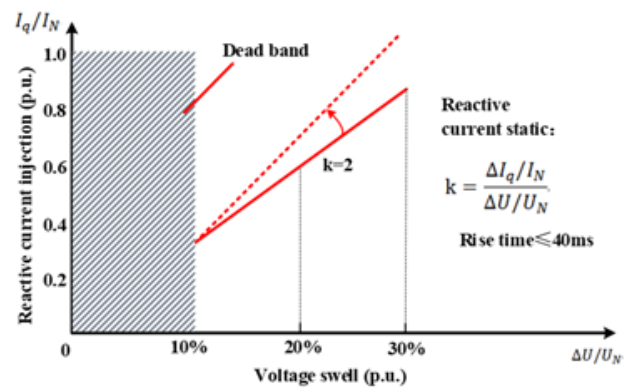

Fig. 3. Reactive current injection requirement of German E. ON Netz code.

The time-domain response characteristics of the WF during grid voltage swells are shown in Fig. 4. In Fig. 4(a), after the grid voltage $V_{\mathrm{g}}$ increases above its threshold $V_{\mathrm{g}}^{\text {thres }}$ at $t_{1}$, WTs supply additional reactive current to support the gird voltage recovery. Note that $V_{\mathrm{g}}^{\text {thres }}=1.1 V_{\mathrm{N}}, V_{N}$ denotes the nominal voltage. Then the WT terminal voltage $V_{\mathrm{W}}$ begins to increase at $t_{1}$ (see Fig. 4(b)). When $\mathrm{V}_{\mathrm{W}}$ is beyond its threshold value at $t_{2}$, the voltage droop control is activated. The reactive output of WTs and WT terminal voltages reach to the balance at $t_{3}$. When the grid swell event is cleared at $t_{4}$, the voltage begins to return to the normal range.

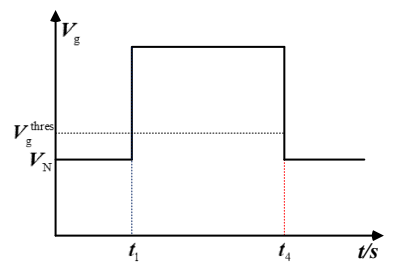

(a) Grid voltage at PCC

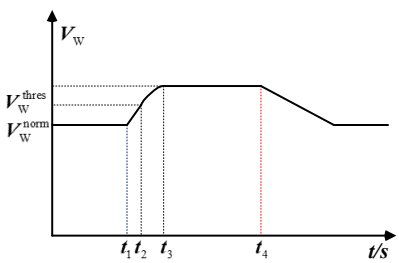

(b) WT terminal voltage
Fig. 4. Schematic response curves of WF for a grid voltage swell

\section{CONTROL ARChitecture AND WF MODELING}

\section{A. Control Concept}

The time-domain process of the WF operation can be divided into three periods, which are the pre-event period, HVRT period, and post-event recovery period. When a grid voltage swells, WTs should take action as soon as possible to respond to the voltage fluctuation. During the HVRT, if WTs remain to receive reactive power references from the central controller of the WF, WTs may fail to ride through the disturbance due to the long calculation time and time delay of the communication system. Therefore, decentralized control such as local droop control shows superiority due to the fast response capability. The diagram of the optimal coordination of droop control and adaptive model predictive control scheme strategy is shown in Fig. 5.

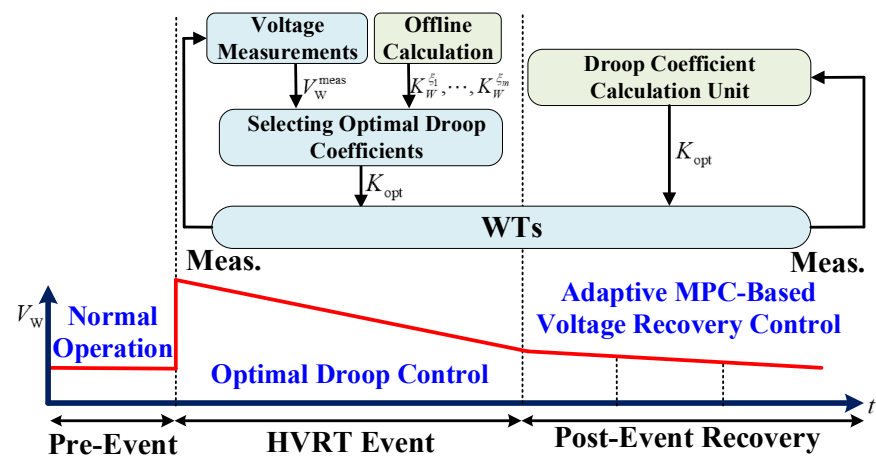

Fig. 5. Diagram of the optimal coordinated voltage control.

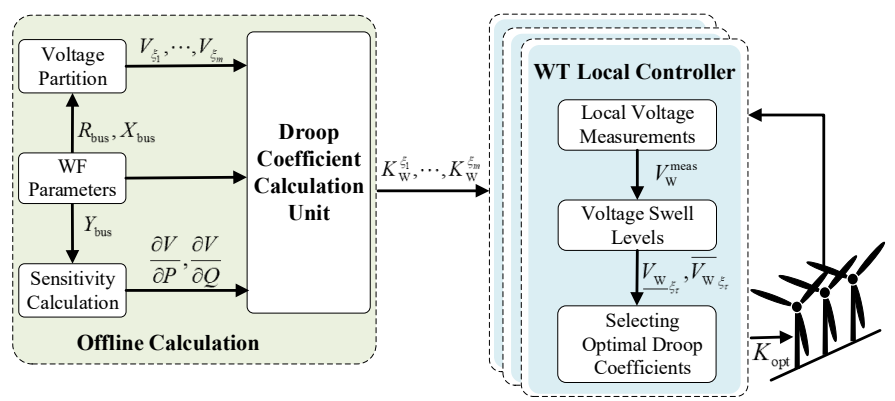

Fig. 6. Diagram of optimal droop control during HVRT.

The framework of the optimal droop control during the HVRT is depicted in Fig. 6. The offline calculation block is designed to pre-calculate the droop coefficient sets of the WTs. The sensitivity calculation block is to calculate the voltage sensitivity coefficients with respect to the active and reactive power output of WTs. The voltage swell partition block is used to divide the voltage swell magnitude into different levels. An optimization problem considering the dynamic behavior of the WT converter is formulated and solved in the WF controller to obtain the optimal droop coefficients of WTs. The droop coefficient is sent to the WT and stored in the WT local controller. During the HVRT, WTs carry out the local droop control by following the optimal droop coefficient to regulate the reactive power output. The optimal droop coefficients are determined by taking the local voltage measurements and voltage swell levels into consideration.

Fig. 7 shows the diagram of adaptive MPC-based voltage recovery control during the post-event recovery period. The MPC algorithm is used to formulate the voltage optimization problem with the droop control. The voltage fluctuations of the WT terminal inside the WF are predicted considering the wind power uncertainty and reactive power injection over the subpredictive horizon $\Delta T_{\mathrm{P}}$. The droop coefficients of WTs are optimized based on initial voltage measurements, voltage predictions as well as voltage sensitivity coefficients during each predictive horizon $T_{\mathrm{P}}$, which is sent to the WT local controllers and remains unchanged until the next optimization period. Each WT regulates the reactive power output by following the optimal droop coefficient at the WT control period $T_{\mathrm{C}}$. Therefore, the reactive power output of WTs can be 
continuously adjusted to fast respond to the WT terminal voltage fluctuations.

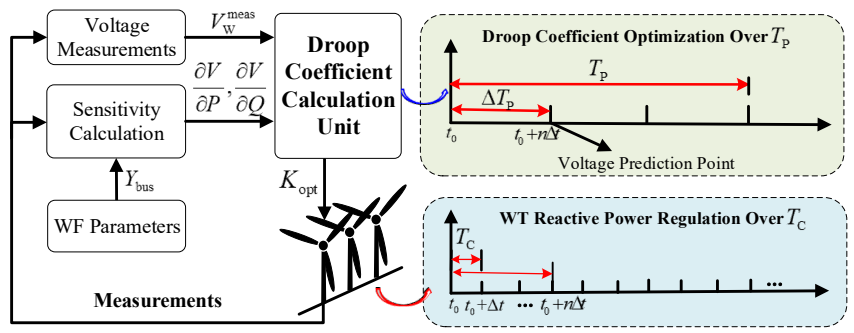

Fig. 7. Diagram of adaptive MPC-based voltage recovery control during the post-event recovery period.

\section{B. Modeling of WTs and WF}

The droop-based reactive power control model is adopted to regulate the WT terminal voltage during the grid voltage swell. The WT searches the reactive power reference by tracking a Q$\mathrm{V}$ droop curve (see in Fig. 8), which can be formulated as,

$$
\begin{aligned}
& Q_{\mathrm{W}}^{\text {ref }}=\left(V_{\mathrm{W}}-V_{\mathrm{N}}\right) K \\
& Q_{\mathrm{W} \text { min }} \leq Q_{\mathrm{W}}^{\text {ref }} \leq Q_{\mathrm{W} \text { max }}
\end{aligned}
$$

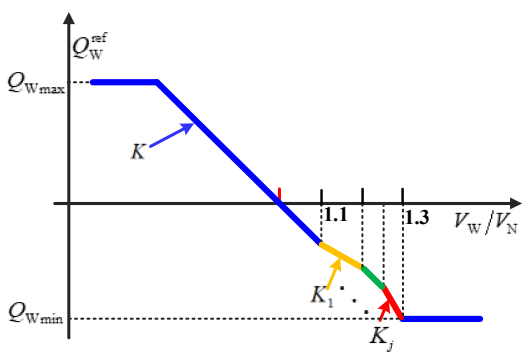

Fig. 8. Droop control curve between voltage and reactive power.

where $Q_{\mathrm{w}}^{\mathrm{ref}}$ denotes the reactive power reference of the WT, $Q_{\mathrm{Wmin}}$ and $Q_{\mathrm{W} \max }$ denote the minimum and maximum available reactive power of the $\mathrm{WT}$, respectively, $V_{\mathrm{W}}$ is the voltage measurement at the WT terminal, $V_{N}$ denotes the nominal voltage, $K$ is the droop coefficient for the normal voltage ranges, as shown in the blue part of the droop characteristic in Fig. 8, and $K_{j}$ is the droop coefficient for the HVRT and post-event recovery periods.

In the detailed WT model, the control diagram of the WT terminal voltage, reactive power, and reactive current is shown in Fig.9.

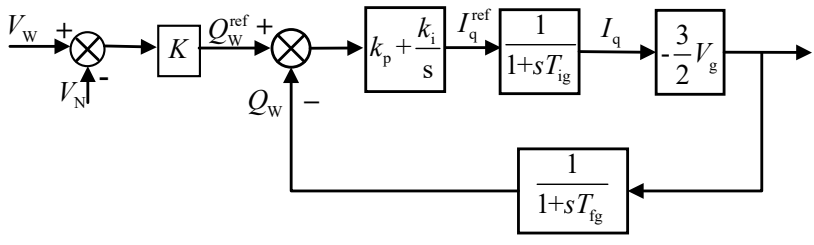

Fig. 9. Control block diagram of WT.

where $V_{\mathrm{g}}$ is the grid voltage amplitude, $k_{\mathrm{p}}$ and $k_{\mathrm{i}}$ are the proportional and integral gains of the PI controller of the outer loop, $T_{\text {ig }}$ is the time constant of the inner loop, and $T_{\text {fg }}$ is the reactive power filter time constant.

Based on [25], the dynamic behavior and time-delay of the reactive power regulation of the WT can be formulated as,

$$
\Delta Q_{\mathrm{W}}=\frac{1}{1+T_{\mathrm{W}} s} \Delta Q_{\mathrm{W}}^{\mathrm{ref}}
$$

where $Q_{\mathrm{W}}$ is the reactive power measurement of the WT. $T_{W}$ is the time constant to consider the dynamic behaviour and time delay of the WTs, which is in the range of 0-10 s. The corresponding continuous-time predictive model of the reactive power output of $n$ WTs can be written as,

$$
\Delta \dot{Q}_{\mathrm{w}}=\boldsymbol{A}_{\mathrm{w}} \Delta Q_{\mathrm{w}}+\boldsymbol{B}_{\mathrm{w}} \Delta Q_{\mathrm{w}}^{\mathrm{ref}}
$$

where

$$
\begin{gathered}
\Delta Q_{\mathrm{W}}=\left[\Delta Q_{\mathrm{W} 1}, \Delta Q_{\mathrm{W} 2}, \ldots, \Delta Q_{\mathrm{W} n}\right]^{T} \\
\Delta Q_{\mathrm{W}}^{\mathrm{ref}}=\left[\Delta Q_{\mathrm{W} 1}^{\mathrm{ref}}, \Delta Q_{\mathrm{W} 2}^{\mathrm{ref}}, \ldots, \Delta Q_{\mathrm{W} n}^{\mathrm{ref}}\right]^{T} \\
\boldsymbol{A}_{\mathrm{W}}=\operatorname{diag}\left(-1 / T_{\mathrm{W} 1},-1 / T_{\mathrm{W} 2}, \ldots,-1 / T_{\mathrm{W} n}\right) \\
\boldsymbol{B}_{\mathrm{W}}=\operatorname{diag}\left(1 / T_{\mathrm{W} 1}, 1 / T_{\mathrm{W} 2}, \ldots, 1 / T_{\mathrm{W} n}\right)
\end{gathered}
$$

Then the continuous state space model of the WF comprised of $n$ WTs can be expressed as,

$$
\left\{\begin{array}{l}
\Delta \dot{x}=\boldsymbol{A} \Delta x+\boldsymbol{B} \Delta u \\
\Delta y=\boldsymbol{C} \Delta x
\end{array}\right.
$$

where

$$
\begin{aligned}
& \Delta x=\left[\Delta Q_{\mathrm{W} 1}, \Delta Q_{\mathrm{W} 2}, \ldots, \Delta Q_{\mathrm{W} n}\right]^{T} \\
& \Delta u=\left[\Delta Q_{\mathrm{W} 1}^{\mathrm{ref}}, \Delta Q_{\mathrm{W} 2}^{\mathrm{ref}}, \ldots, \Delta Q_{\mathrm{W} n}^{\mathrm{ref}}\right]^{T} \\
& \Delta y=\left[\Delta Q_{\mathrm{W} 1}, \Delta Q_{\mathrm{W} 2}, \ldots, \Delta Q_{\mathrm{W} n}\right]^{T} \\
& \boldsymbol{A}=\boldsymbol{A}_{\mathrm{W}}, \boldsymbol{B}=\boldsymbol{B}_{\mathrm{W}}, \boldsymbol{C}=\boldsymbol{I}_{N_{\mathrm{W}}}=\boldsymbol{I}^{n \times n}
\end{aligned}
$$

Based on (5), the discrete time state-space model with sampling time $\Delta T_{\mathrm{S}}$ can be derived from the continuous model as,

$$
\Delta x(k+1)=\boldsymbol{M} \Delta x(k)+\boldsymbol{N} \Delta u(k)
$$

$$
\Delta y(k)=\boldsymbol{C} \Delta x(k)
$$

where

$$
\boldsymbol{M}=e^{A \Delta T_{\mathrm{S}}}, \quad \boldsymbol{N}=\int_{0}^{\Delta T_{\mathrm{S}}} e^{A t} \boldsymbol{B} d t
$$

\section{Proposed Optimal Combined Control Strategy}

The optimal droop control and adaptive MPC-based voltage recovery control (AMVRC) are implemented for fast WT terminal voltage reduction during the HVRT and post-event voltage restoration, respectively. The voltage regulation is achieved by tracking the reactive power references of each WT, which are determined by the optimal droop coefficients and local voltage measurements.

\section{A. Optimal Droop Coefficient Calculation}

Since the response time of WTs during the HVRT is short according to the requirement of the grid code, the optimal droop control scheme is developed to achieve a fast reactive power sharing among WTs by following the optimized droop coefficients. Based on (1), the incremental reactive power model with the droop control can be formulated as,

$$
\Delta Q_{\mathrm{W}_{j}}^{\mathrm{ref}}=\left(V_{\mathrm{W} j}-V_{\mathrm{N}}\right) K_{j}-Q_{\mathrm{W}_{j}}^{0}
$$

where $Q_{\mathrm{W} j}^{0}$ denote initial reactive power measurement.

With conventional droop control, the droop coefficients of WTs are identical, which cannot ensure the global optimality of the WF operation during the HVRT period. Therefore, the droop coefficient optimization algorithm is developed to 
improve the HVRT capability and guarantee the global optimality of the WF operation. Based on (7), the relationship between $\Delta u(k)$ and the decision variables $K_{\mathrm{W}}$ can be expressed as,

$$
\Delta u(k)=\mu_{\mathrm{W}}(k) K_{\mathrm{W}}+F(k)
$$

with

$$
\begin{aligned}
& K_{\mathrm{W}}=\left[K_{\mathrm{W} 1}, K_{\mathrm{W} 2}, \cdots, K_{\mathrm{Wn}}\right]^{T} \\
& \boldsymbol{F}(k)=\left[-Q_{\mathrm{W} 1}^{0},-Q_{\mathrm{W} 2}^{0}, \cdots,-Q_{\mathrm{W} n}^{0}\right]^{T} \\
& \boldsymbol{\mu}_{\mathrm{W}}(k)=\left[\begin{array}{llll}
V_{\mathrm{w} 1}^{\mathrm{pre}}(k)-V_{\mathrm{N}} & & & \\
& V_{\mathrm{W} 2}^{\mathrm{pre}}(k)-V_{\mathrm{N}} & \ddots & \\
& & & V_{\mathrm{W} n}^{\mathrm{pre}}(k)-V_{\mathrm{N}}
\end{array}\right]
\end{aligned}
$$

where $V_{\mathrm{W}}^{\mathrm{pre}}$ is the predictive voltage of the WT at the event occurrence, which is determined based on the measurement information.

Inserting (8) into (6), the discrete state-space model of the WF with droop control can be rewritten as,

$$
\begin{aligned}
\Delta x(k+1) & =\boldsymbol{M} \Delta x(k)+\boldsymbol{N} \boldsymbol{\mu}_{\mathrm{W}}(k) K_{\mathrm{W}}+\boldsymbol{N} \boldsymbol{F}(k) \\
\Delta y(k) & =\boldsymbol{C} \Delta x(k)
\end{aligned}
$$

In order to facilitate the fast response of the WTs during the HVRT, the control objective is to minimize the terminal voltage deviations of WTs, which can be formulated as,

$$
\operatorname{Obj}=\min \left(\sum_{j=1}^{n}\left\|V_{\mathrm{W}_{j}}^{\mathrm{pre}}-V_{\mathrm{N}}+\frac{\partial V_{\mathrm{W} j}}{\partial P_{\mathrm{W}}} \Delta P_{\mathrm{W}}+\frac{\partial V_{\mathrm{W} j}}{\partial Q_{\mathrm{W}}} \Delta Q_{\mathrm{W}}\right\|^{2}\right)
$$

with

$$
\begin{aligned}
& \frac{\partial V_{\mathrm{W} j}}{\partial P_{\mathrm{W}}}=\left[\frac{\partial V_{\mathrm{W} j}}{\partial P_{\mathrm{W} 1}}, \frac{\partial V_{\mathrm{W} j}}{\partial P_{\mathrm{W} 2}}, \cdots, \frac{\partial V_{\mathrm{W} j}}{\partial P_{\mathrm{W} j}}, \cdots, \frac{\partial V_{\mathrm{W} j}}{\partial P_{\mathrm{W} n}}\right] \\
& \frac{\partial V_{\mathrm{W} j}}{\partial Q_{\mathrm{W}}}=\left[\frac{\partial V_{\mathrm{W} j}}{\partial Q_{\mathrm{W} 1}}, \frac{\partial V_{\mathrm{W}_{j}}}{\partial Q_{\mathrm{W} 2}}, \cdots, \frac{\partial V_{\mathrm{W} j}}{\partial Q_{\mathrm{W} j}}, \cdots, \frac{\partial V_{\mathrm{w}_{j}}}{\partial Q_{\mathrm{W} n}}\right]
\end{aligned}
$$

where $\frac{\partial V_{\mathrm{W} j}}{\partial P_{\mathrm{W}}}$ and $\frac{\partial V_{\mathrm{W} j}}{\partial Q_{\mathrm{W}}}$ are voltage sensitivity coefficient matrices with respect to the active power and reactive power injection of WTs, respectively.

Based on (1), the reactive power references of WTs are related to the terminal voltage measurements of WTs and the droop coefficients. Hence, the constraint of the droop coefficient $K_{j}$ is to guarantee that the reactive power of WTs will not violate the available reactive power limits,

$$
\begin{aligned}
& \frac{Q_{\mathrm{W} j \text { min }}}{V_{\mathrm{W} j}^{\text {pre }}-V_{\mathrm{N}}} \leq K_{j} \leq \frac{Q_{\mathrm{W} j \text { max }}}{V_{\mathrm{W} j}^{\text {pre }}-V_{\mathrm{N}}} \\
& \frac{2 Q_{\mathrm{W} j}}{3 V_{\mathrm{W}}} \geq \alpha\left(V_{\mathrm{W}} / V_{\mathrm{N}}-1\right) \times I_{\mathrm{N}}
\end{aligned}
$$

where $I_{\mathrm{N}}$ is the rated current of the WT; and $\alpha$ is an adjustable coefficient, which should be no less than 2 .

Equations (12) show the constraint for the reactive current injection of the WT [26]. The formulated optimization problem can be transformed into a standard quadratic programming (QP) problem, which can be efficiently solved by commercial QP solvers in milliseconds [18].

\section{B. Optimal Droop Control During HVRT}

WTs inside the WF may suffer from different voltage swell conditions due to the complexity and uncertainty of actual conditions, the optimal droop coefficients should be calculated by considering various event severity. In the control period, the optimized droop coefficients applied for different voltage swell levels are stored in local controllers of each WT. Once the voltage swell event occurs, each WT local controller can fast respond to the terminal voltage fluctuations by tracking the stored optimal droop coefficient based on its local voltage measurements.

The voltage difference resulted from the topology of the WF collection system is a non-negligible factor during the event period. Let $\mathbb{C}$ denotes the set of complex numbers. $s_{i}=p_{i}+$ $j q_{i} \in \mathbb{C}$ denotes the complex power injection at bus $i$. Define bus 0 as a feeder bus and the other buses in $N_{0}$ as branch buses, where bus $j$ is the bus with the highest level of voltage swell. The voltage of bus 0 and bus $j$ are denoted as $v_{0}$ and $V_{\text {ser }}$, respectively. Based on the linear DistFlow model [27], the voltage difference can be formulated as,

$$
v=X q+R p+\mathrm{I} v_{0}
$$

with

$$
X_{i j}=2 \sum_{(h, k) \in L_{i} \cap L_{j}} x_{h k}, R_{i j}=2 \sum_{(h, k) \in L_{i} \cap L_{j}} r_{h k}
$$

where $v=\left[V_{\mathrm{W} 1}, V_{\mathrm{W} 2}, \cdots, V_{\mathrm{ser}}, \cdots, V_{\mathrm{W} i}, \cdots, V_{\mathrm{W} n}\right]^{T}, \quad V_{\mathrm{W} i} \in \mathrm{R}_{+}$ denotes the squared voltage magnitude of the bus $i . p=$ $\left[P_{\mathrm{W} 1}, P_{\mathrm{W} 2}, \cdots, P_{\mathrm{W} n}\right]^{T}$ and $q=\left[Q_{\mathrm{W} 1}, Q_{\mathrm{W} 2}, \cdots, Q_{\mathrm{W} n}\right]^{T}$ denote the active power injection and reactive power injection, respectively. $r_{h k}$ and $x_{h k}$ are the resistance and reactance of the line segment between bus $h$ and bus $k . L_{i} \subseteq \mathcal{R}$ is the set of edges in the path from the bus 0 to bus $i, \mathcal{R} \in N_{0} \times N_{0}$. Note that $V_{\text {ser }}$ is the measurement value and $v_{0}$ is unknown.

Define the voltage swell level $V_{\xi_{\tau}}=\left[\underline{V}_{\xi_{\tau}}, \bar{V}_{\xi_{\tau}}\right]$ as per 0.01 p.u., $\tau=\{1,2, \ldots, m\}$, note that $\underline{V}_{\xi_{1}}=1.1 p . u$. and $\bar{V}_{\xi_{m}}=$ 1.3p.u. according to the grid code requirement for HVRT capability during the grid voltage swell in Fig. 7. Define the optimal droop coefficient set $K_{W}=\left\{K_{\mathrm{W}}^{\xi_{1}}, K_{\mathrm{W}}^{\xi_{2}}, \ldots, K_{\mathrm{W}}^{\xi_{\tau}} \ldots, K_{\mathrm{W}}^{\xi_{m}}\right\}$, the relation between the voltage swell level and the optimal droop coefficient can be formulated as,

$$
\begin{aligned}
& \text { If } V_{\text {ser }} \in\left[\underline{V}_{\xi_{\tau}}, \bar{V}_{\xi_{\tau}}\right] \\
& {\underline{\mathrm{W}_{\xi}}}_{\xi_{\tau}}=\left\{V_{\mathrm{W} 1} \xi_{\tau}, V_{\mathrm{W} 2}, \cdots, \underline{V_{\mathrm{W} i}} \bar{\xi}_{\tau}, \cdots, \underline{V_{\mathrm{W} n} \xi_{\tau}}\right\} \\
& \overline{V_{\mathrm{W}} \xi_{\tau}}=\left\{\overline{V_{\mathrm{W} 1} \xi_{\tau}}, \overline{V_{\mathrm{W} 2} \xi_{\tau}}, \cdots, \overline{V_{\mathrm{W} i}}, \cdots, \overline{V_{\mathrm{W} n} \xi_{\tau}}\right\} \\
& K_{\mathrm{W}}=K_{\mathrm{W}}^{\xi_{\tau}}=\left\{K_{\mathrm{W} 1}^{\xi_{\tau}}, K_{\mathrm{W} 2}^{\xi_{\tau}}, \cdots, K_{\mathrm{W} i}^{\xi_{\tau}}, \cdots, K_{\mathrm{W} n}^{\xi_{\tau}}\right\}
\end{aligned}
$$

where $V_{\xi_{\tau}}$ and $\overline{V_{\xi_{\tau}}}$ denote the lower and upper limits of the voltage swell level, ${\underline{V_{\mathrm{W}}}}_{\xi_{\tau}}$ and ${\overline{V_{\mathrm{W}}}}_{\xi_{\tau}}$ denote the lower and upper limit sets of WTs terminal voltage under different event conditions, and $K_{\mathrm{W} j}^{\xi_{\tau}}$ denote the optimal droop coefficient of $j$ th WT at the voltage swell level $V_{\xi_{\tau}}$. The lower and upper limit of each WT terminal voltage in (16) can be calculated based on (14) and (15).

The control action of each WT fully depends on the local measurements when the grid voltage swell occurs. When an 
actual event occurs, the initial terminal voltage $V_{\text {ser }}$ of the bus $j$ can be measured. If the terminal voltage measurement $V_{\mathrm{W} i}^{\text {meas }} \in$ $\left[V_{\mathrm{W} i}, \overline{V_{\mathrm{W} i} \xi_{\tau}}\right]$, the optimal droop coefficient set of WTs can be determined in the appropriate voltage swell level based on (16) and (17), then the reactive power reference of each WT can be sent to each WT local controllers.

\section{Adaptive MPC-Based Voltage Recovery Control During Post-Event Recovery Period}

During the post-event recovery period, the response time of WTs is longer than that during the HVRT. The AMVRC scheme is implemented to achieve an excellent post-event voltage restoration performance. Each WT regulates the reactive power by following the optimal droop coefficient, which is circularly updated with the voltage sensitivity coefficient and voltage measurements.

In the AMVRC scheme, $\mu_{\mathrm{W}}(k)$ in (8) should be updated in each sub-predictive horizon $\Delta T_{\mathrm{P}}$ with the voltage change. The coefficients of the droop curves are only optimized once in each predictive horizon with the initial voltage measurements. Since the accurate voltage measurements are not available during this period, the voltage conditions of WTs can be predicted by using the sensitivity coefficients. Based on (8), for the $k t h$ predictive step, $\mu_{\mathrm{W}}(k)$ can be expressed as,

$$
\begin{aligned}
\boldsymbol{\mu}_{\mathrm{W}}(k) & =\operatorname{diag}\left[\sigma_{\mathrm{Q}} \Delta y(k)+\sigma_{\mathrm{P}} \Delta \boldsymbol{P}_{\mathrm{W}}(k)+V_{\mathrm{W}}^{0}-V_{\mathrm{ref}}\right] \\
& =\operatorname{diag}\left[\sigma_{\mathrm{Q}} \boldsymbol{C} \Delta x(k)+\sigma_{\mathrm{P}} \Delta \boldsymbol{P}_{\mathrm{W}}(k)+V_{\mathrm{W}}^{0}-V_{\mathrm{ref}}\right] \\
& =\operatorname{diag}\left\{\sigma _ { \mathrm { Q } } \boldsymbol { C } \left[\boldsymbol{M} \Delta x(k-1)+\boldsymbol{N} \boldsymbol{\mu}_{\mathrm{W}}(k-1) K_{\mathrm{W}}\right.\right. \\
& \left.+\boldsymbol{N} \boldsymbol{F}(k-1)]+\sigma_{\mathrm{P}} \Delta \boldsymbol{P}_{\mathrm{W}}(k)+V_{\mathrm{W}}^{0}-V_{\mathrm{ref}}\right\}
\end{aligned}
$$

where $\sigma_{\mathrm{P}}$ and $\sigma_{\mathrm{Q}}$ are the voltage sensitivity coefficient matrices with respect to the active and reactive power injection of WTs, respectively. $\Delta P_{\mathrm{W}}(k)$ is the incremental vector of the active power $\Delta P_{\mathrm{W} j}$ at the $k$ th predictive step, $\Delta P_{\mathrm{W} j}(k)=P_{\mathrm{W} j}(k)-$ $P_{\mathrm{W} j}^{0}$. In (22), $\mu_{\mathrm{W}}(k)$ can be deduced back to the initial state space matrix. Nevertheless, $\Delta u(k)$ comprises a polynomial of $K_{\mathrm{W}}$ which cannot be formulated to a Quadratic Programming (QP) problem. So the MPC-based discrete state space model should be reformulated.

Define the following equation:

$$
\left\{\begin{array}{l}
\operatorname{diag}\left\{\left[\sigma_{\mathrm{Q}} \boldsymbol{C} \boldsymbol{N} \boldsymbol{\mu}_{\mathrm{W}}(k-1) K_{\mathrm{W}}\right] K_{\mathrm{W}}\right\} \approx \boldsymbol{\Pi}(k-1) \\
\operatorname{diag}\left[\sigma_{\mathrm{Q}} \boldsymbol{C N} \boldsymbol{F}(k-1)+\sigma_{\mathrm{P}} \Delta \boldsymbol{P}_{\mathrm{W}}(k)+V_{\mathrm{W}}^{0}-V_{\text {ref }}\right]=\boldsymbol{\Lambda}(k-1)
\end{array}\right.
$$

With the Taylor series expansion near the operating point, the polynomial of $K_{\mathrm{w}}$ can be degraded and linearized as,

$$
\boldsymbol{\Pi}(k-1) \approx \boldsymbol{\Gamma}(k-1) K_{\mathrm{W}}+\Upsilon(k-1)
$$

where $\Gamma(k-1)$ and $\Upsilon(k-1)$ are the coefficient matrices related to the Taylor series expansion. Substituting (22)-(24) into (9), the MPC-based discrete state-space WF model with local droop control should be linearized as,

$$
\begin{gathered}
\Delta x(k+1)=\boldsymbol{\rho}(k) K_{\mathrm{W}}+\boldsymbol{f}(k) \\
\Delta y(k)=\boldsymbol{C} \Delta x(k)
\end{gathered}
$$

where

$$
\begin{gathered}
\boldsymbol{\rho}(k)=\boldsymbol{M} \rho(k-1)+\boldsymbol{N} \Gamma(k)+\boldsymbol{N} \Lambda(k) \\
\boldsymbol{f}(k)=\boldsymbol{M} f(k-1)+\boldsymbol{N} \Upsilon(k)+\boldsymbol{N} F(k) \\
\forall k \in 1,2, \cdots, N_{\mathrm{P}}
\end{gathered}
$$

$$
\rho(0)=N \mu_{\mathrm{W}}(0), \boldsymbol{f}(0)=\boldsymbol{N F}(0)
$$

Compared with the HVRT control, the initial voltage of the WTs terminal is obtained from the measurements. Meanwhile, the objective function of the post-event recovery period is taken voltage prediction $V_{\mathrm{S}}^{\text {pre }}$ of the external grid into account, which can be formulated as,

$$
\begin{gathered}
\mathrm{Obj}=\min \left(\sum_{k=1}^{N_{p}} \sum_{j=1}^{n}\left\|\Delta V_{\mathrm{W} j}(k)\right\|^{2}\right) \\
\Delta V_{\mathrm{W} j}=V_{\mathrm{W}}^{0}-V_{N}+\frac{\partial V_{\mathrm{W} j}}{\partial P_{\mathrm{W}}} \Delta P_{\mathrm{W}}+\frac{\partial V_{\mathrm{W} j}}{\partial Q_{\mathrm{W}}} \Delta Q_{\mathrm{W}}+\frac{\partial V_{\mathrm{W} j}}{\partial V_{\mathrm{s}}} V_{\mathrm{s}}^{\text {pre }}
\end{gathered}
$$

where $\frac{\partial V_{\mathrm{W} j}}{\partial V_{\mathrm{S}}}$ is the voltage sensitivity coefficient matrix with respect to the external grid voltage. The voltage prediction value $V_{\mathrm{S}}^{\text {pre }}$ of the external grid can be obtained from the phasor measurement unit (PMU), which is usually used to measure the transient voltage vector.

According to the grid code on the HVRT time-voltage profile of grid-connected WTs, the magnitude and time of WT terminal voltage are subjected to the following constraints,

$$
\begin{gathered}
\frac{Q_{j \min }}{V_{\mathrm{W} j}-V_{\mathrm{N}}} \leq K_{j} \leq \frac{Q_{j \max }}{V_{\mathrm{W} j}-V_{\mathrm{N}}} \\
\frac{2 Q_{\mathrm{W} j}}{3 V_{\mathrm{W}}} \geq \alpha\left(V_{\mathrm{W}} / V_{\mathrm{N}}-1\right) \times I_{\mathrm{N}} \\
V_{\mathrm{W} / T_{\mathrm{ref}}=0.5 s} \leq 1.3 p . u . \\
V_{\mathrm{W} / T_{\mathrm{ref}}=1 s} \leq 1.25 p . u . \\
V_{\mathrm{W} / T_{\mathrm{ref}}=10 s} \leq 1.2 p . u .
\end{gathered}
$$

\section{CASe Study}

\section{A. Test System}

A WF model consisting of $32 \times 5 \mathrm{MW}$ DFIG-based WTs shown in Fig. 1, is established in Matlab/Simulink to verify the effectiveness of the proposed control scheme. The wind field model considering the turbulences and wake effects for the WF is generated using the Sim-WindFarm, a toolbox for dynamic wind farm modeling and simulation [28]. The test system parameters of the WF are shown in Table I. Taking the voltage swell event with the PCC voltage of 1.25 p.u. as an example, the HVRT threshold $V_{\text {th }}$ of all WTs can be calculated in Table II. It can be seen that the HVRT thresholds of all WTs are different, which are related to their electrical distances from the PCC and their operating condition.

The control performances of the proposed optimal coordination control scheme are verified by comparing to two other schemes, which are the conventional PI-based control scheme (PI) and the MPC-based enhanced voltage control scheme (MPC) [18]. The control action of the MPC scheme is carried out every second and the PI control scheme is carried out every $2 \mathrm{~s}$. In the PI control scheme, the reactive power references of the WTs are dispatched to the WTs based on a proportional distribution control scheme. The PI controller parameters are designed as $k_{\mathrm{p}}=10$ and $k_{\mathrm{i}}=100$ [29]. In the proposed control scheme, the control action during the pre- 
event period is carried out every $2 \mathrm{~s}$ by using the PI scheme for the normal operation, and the optimal droop coefficients are generated. The ADVRC scheme during the post-event recovery period is carried out every $2 \mathrm{~s}\left(T_{\mathrm{P}}=2 \mathrm{~s}\right)$ to generate the optimal droop coefficients. The WTs regulate the reactive power output every $0.1 \mathrm{~s}\left(T_{\mathrm{C}}=0.1 \mathrm{~s}\right)$ based on the droop coefficients.

TABLE I

TEST SYSTEM PARAMETERS

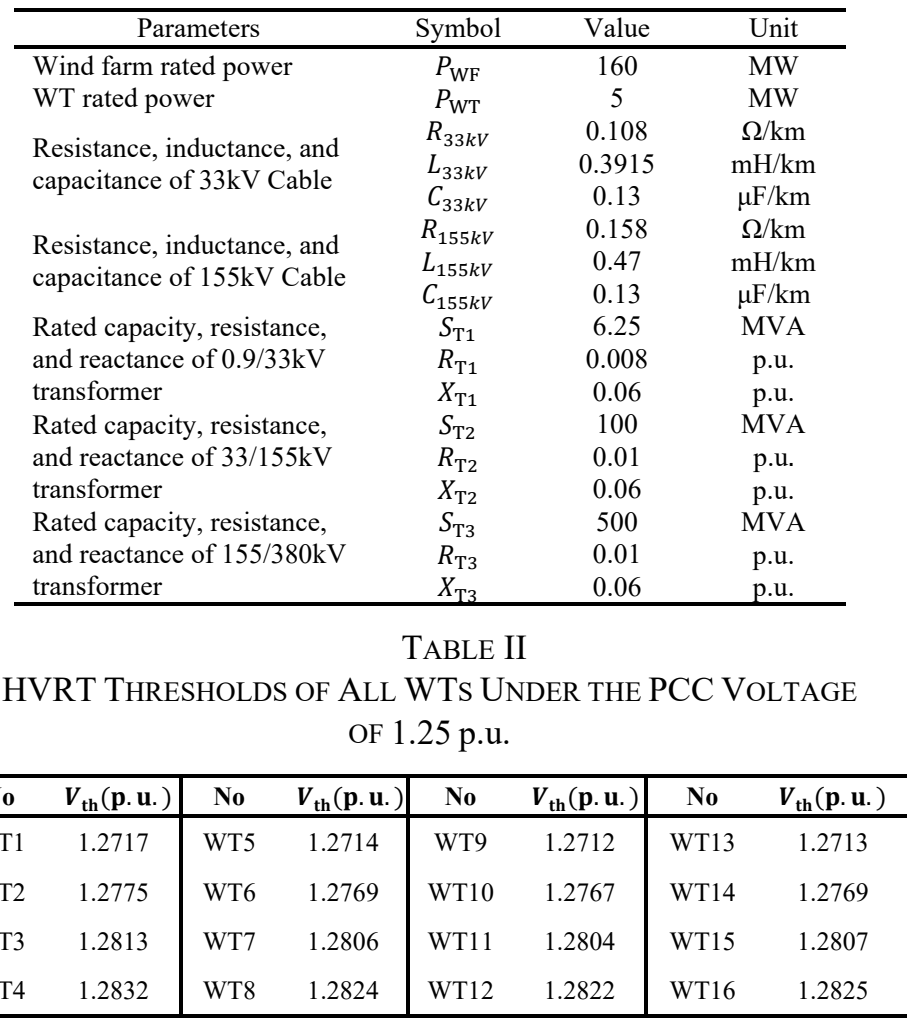

\section{B. Control Performance Under Different Schemes}

In this simulation, the voltage swell starts at $t=20.1 \mathrm{~s}$ and last for $0.5 \mathrm{~s}$. The simulation time is $40 \mathrm{~s}$. The comparative result and analysis between the three control schemes are shown in Figs. 10-19.

The wind speed of the different WTs and the active power outputs of the WF and WT8 are shown in Figs. 10-11. Since WTs operate in the maximum power point tracking (MPPT) mode, the active power output of each WT is the same. Besides, the total active power output of the WF tracks the available wind power during the whole simulation period. In Fig. 10(a), the maximum active power output is approximately $108 \mathrm{MW}$, which is about $67.5 \%$ of $\mathrm{P}_{\mathrm{N}}$.

The voltage profiles of WTs with the three control schemes are shown in Figs. 12-14. It is found that the proposed control scheme shows better performance during HVRT than those with the conventional PI control and MPC control schemes. During the post-event voltage recovery period, the proposed control scheme and MPC control schemes show superiority compared to those with the PI control scheme, since the WT terminal voltage recovers within the feasible ranges more quickly than the PI control. During 20.1 20.3 s, the event results in a sudden increase of the WT terminal voltage, which violates their threshold value. For the proposed control scheme, the WT terminal voltage decrease below 1.25 p.u. less than $0.2 \mathrm{~s}$ after the event occurrence (see Fig. 12b) and the post-event recovery is faster (see Fig. 12a). For the PI control and MPC control schemes, the WT terminal voltage decrease below 1.25 p.u. more than $0.5 \mathrm{~s}$ after the event occurrence (see Figs. 13 and $14 \mathrm{~b}$ ). For the PI control scheme, the post-event recovery is slow. $V_{\mathrm{W}}$ goes back to its threshold value slowly after the event occurrence. The simulation result indicates the proposed control scheme can achieve more effective and faster reactive power support of WF by optimal droop control, and the post-event recovery process is faster.

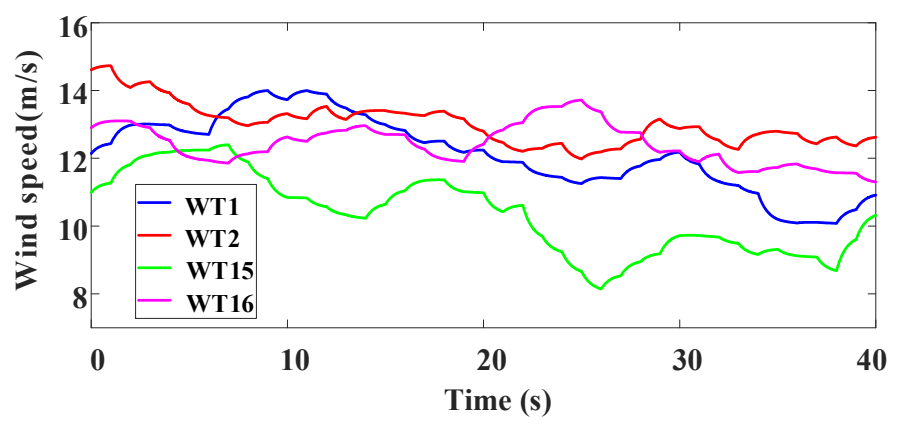

Fig.10. Wind speed of the WTs.

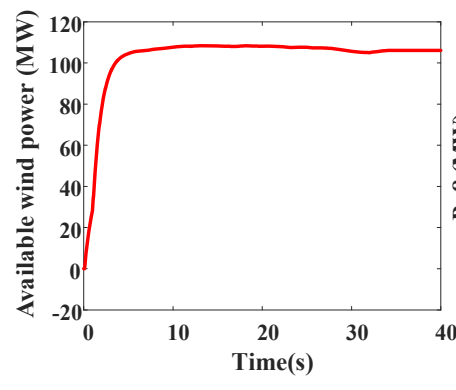

(a)

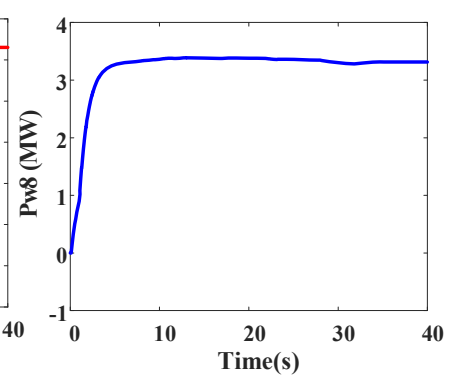

(b)
Fig.11. Active power output. (a) WF, (b) WT8.

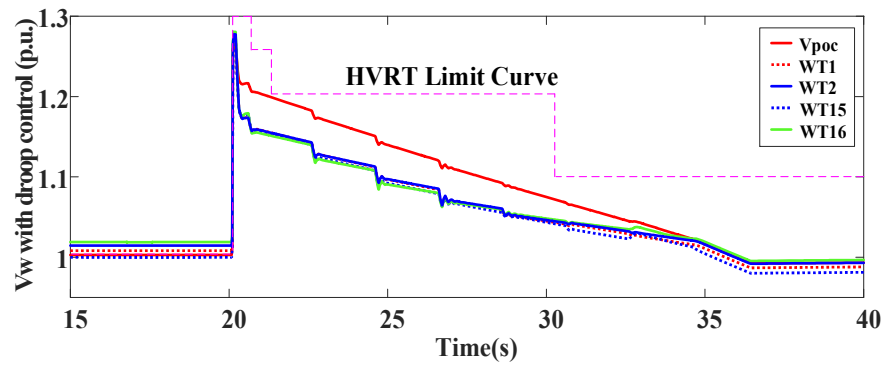

(a)

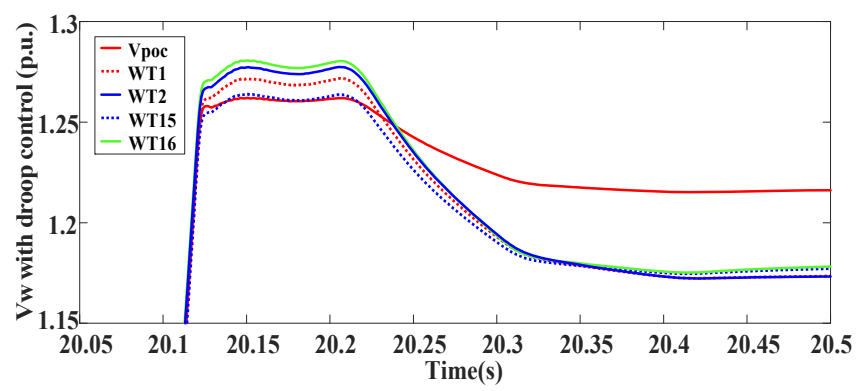

(b)

Fig. 12. Voltage profile of WTs with the proposed control scheme, (a) zoomed part in the voltage axis, (b) zoomed part in the time axis. 


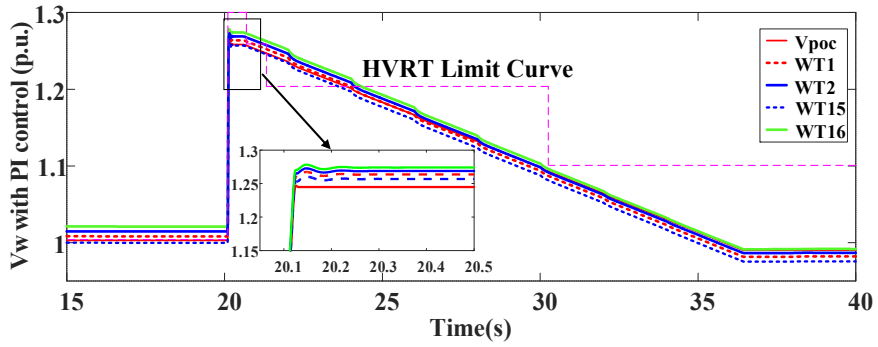

Fig. 13. Voltage profile of WTs with PI control scheme.

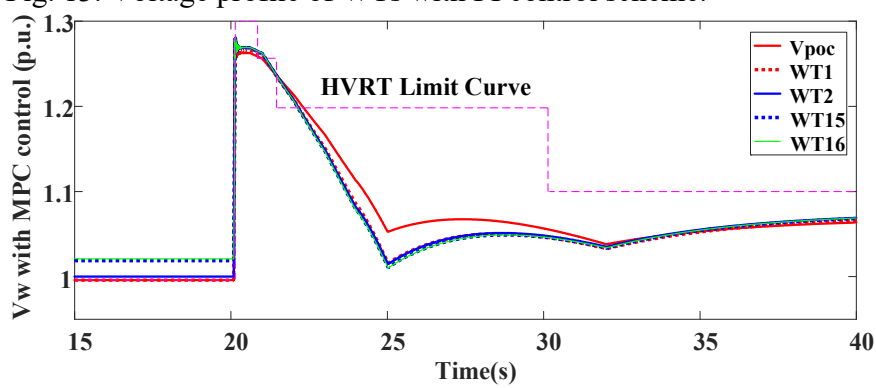

(a)

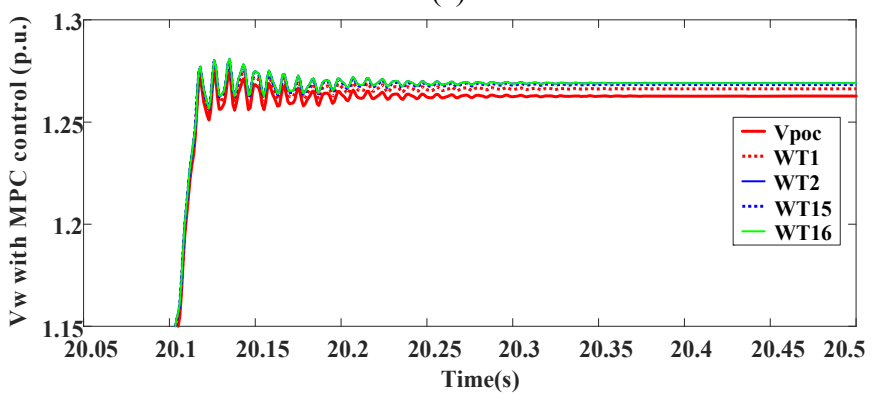

(b)

Fig. 14. Voltage profile of WTs with MPC scheme, (a) zoomed part in the voltage axis, (b) zoomed part in the time axis.

Fig. 15 shows the voltage performances of WT8 with the three control schemes. WT8 is the WT at the end of the first feeder, which has the highest risk to violate the voltage thresholds under grid voltage swell conditions. The voltage of the WT8 terminal bus with the proposed scheme shows superiority compared to those with the PI and MPC control schemes. From $0 \mathrm{~s}$ to $20.1 \mathrm{~s}$, the voltages with the three control schemes are similar, which are kept at around 1.02 p.u. After $20.1 \mathrm{~s}$, the voltage rises to about 1.272 p.u. with the reactive power output increasing while the voltage with the proposed scheme increasing to approximately 1.283 p.u.. The voltage with the proposed and MPC control schemes decrease to 1.1 p.u. less than $4.2 \mathrm{~s}$ after the event occurrence while those with the PI control scheme needs more than $10 \mathrm{~s}$ after the event occurrence. Fig. 16 shows the reactive power output of the WT8. When the event occurs at $20.1 \mathrm{~s}$, the reactive output of the WT with the proposed control and MPC schemes is maximized, which can reach 3.618 MVar and 4.026 MVar respectively, with an approximate difference of 3.1 MVar and 3.6 MVar compared to the PI control scheme.

Fig. 17 shows the total voltage deviation from the rated voltage of the WF. The overall voltage performance with the proposed scheme shows superiority compared to those with the conventional PI and MPC control schemes. During the HVRT, the overall voltage with the proposed control scheme is better than those with the PI and MPC schemes, which has about 2 p.u. difference.

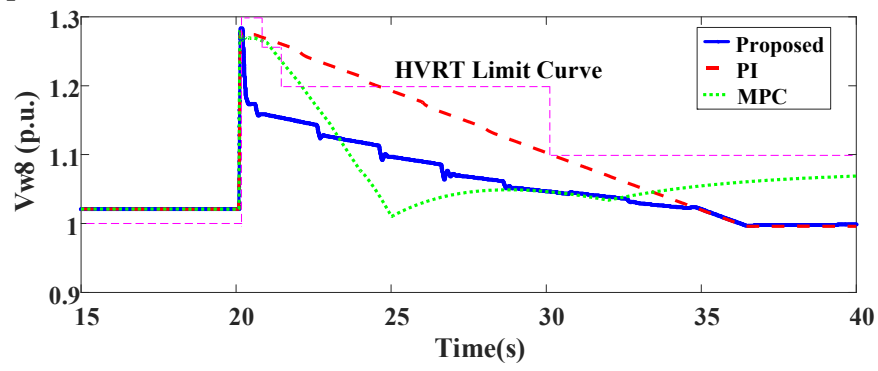

Fig. 15. Voltage profile of WT8.

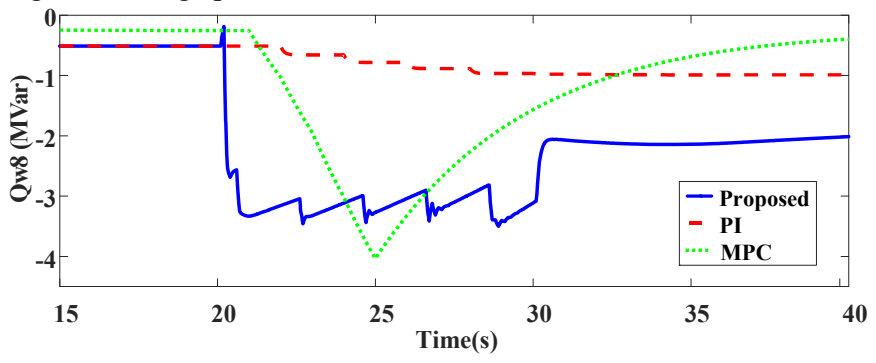

Fig. 16. Reactive power output of WT8.

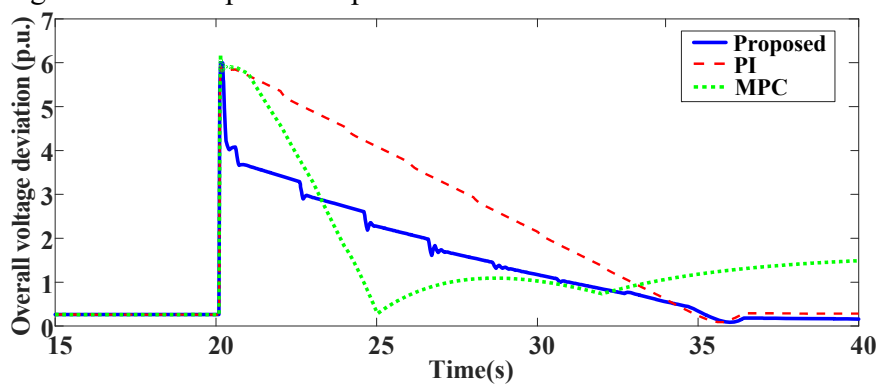

Fig. 17. Overall voltage deviation of WF.

TABLE III

Comparative Analysis of Time Duration of Three Schemes

\begin{tabular}{|c|c|c|c|c|}
\hline \multirow{2}{*}{ Schemes } & \multirow{2}{*}{$V \operatorname{pcc}($ p. u. $)$} & \multicolumn{3}{|c|}{$V$ w(p.u. $)$} \\
\hline & & 1.25 & 1.2 & 1.1 \\
\hline \multirow{3}{*}{ Proposed } & 1.3 & $0.178 \mathrm{~s}$ & $0.974 \mathrm{~s}$ & $8.512 \mathrm{~s}$ \\
\hline & 1.25 & $0.14 \mathrm{~s}$ & $0.194 \mathrm{~s}$ & 4.732 \\
\hline & 1.2 & - & $0.146 \mathrm{~s}$ & 2.526 \\
\hline \multirow{3}{*}{ PI } & 1.3 & $4.338 \mathrm{~s}$ & $7.084 \mathrm{~s}$ & 12.419 \\
\hline & 1.25 & $1.927 \mathrm{~s}$ & $4.002 \mathrm{~s}$ & 8.837 \\
\hline & 1.2 & - & $1.935 \mathrm{~s}$ & 7.442 \\
\hline \multirow{3}{*}{ MPC } & 1.3 & $2.084 \mathrm{~s}$ & $2.961 \mathrm{~s}$ & 4.816 \\
\hline & 1.25 & $1.127 \mathrm{~s}$ & $2.008 \mathrm{~s}$ & 3.612 \\
\hline & 1.2 & - & $1.804 \mathrm{~s}$ & 3.560 \\
\hline \multicolumn{2}{|c|}{ Grid Code } & $\leq 0.5 \mathrm{~s}$ & $\leq 1.0 \mathrm{~s}$ & $\leq 10 \mathrm{~s}$ \\
\hline
\end{tabular}

Table III illustrates the comparative results of the three control schemes under three voltage swell events with the PCC voltage of 1.3 p.u., 1.25 p.u. and 1.2 p.u., respectively. It can be seen that all the time durations with the proposed control scheme are shorter than those with the PI control scheme under the same voltage swell levels. Furthermore, the WT terminal voltage is maintained within the acceptable range and can quickly recover to its normal value after the event clearance. Specifically, all the time duration with the proposed control scheme during the HVRT and post-event recovery process meets specifications of the grid codes in case of any grid voltage swell. In contrast, the time duration with the PI and MPC control schemes during the HVRT period exceeds the 
specified time thresholds of the grid code requirements. For the post-event recovery process, the PI and MPC control schemes meet the time requirements under the lower level of the grid voltage swell. Therefore, the proposed control scheme shows excellent performance for different voltage swell levels.

Fig. 18 shows the voltage profile and reactive power output of WT8 with the three control schemes under subsequent HVRT and LVRT events. As can be seen from Fig. 17(a), the voltage of the WT8 terminal bus with the proposed schemes shows superiority compared to those with the PI and MPC control schemes. when the grid voltage dip event occurs at $\mathrm{t}=19.8 \mathrm{~s}$, the WT terminal voltage decreases to about 0.6 p.u.. From $19.8 \mathrm{~s}$ to $22.8 \mathrm{~s}$, the voltage with the proposed control scheme recovers within the feasible ranges more quickly than those with the PI and MPC control schemes. For the three control schemes, the controllers switch to the normal mode at about $\mathrm{t}=23 \mathrm{~s}$ and keep stable in the remaining period. After $30.1 \mathrm{~s}$, the voltage rises to about $1.252 \mathrm{p}$.u. with the reactive power output increasing. There is an approximate difference of 0.118 p.u. between the proposed control scheme and two other control schemes at $t=30.6 \mathrm{~s}$. As can be seen from Fig. 17(b), during the LVRT and HVRT, the maximum reactive power output of the WT with the proposed control scheme can reach 3.328 MVar and 3.618 MVar, with an approximate difference of 3.1 MVar and 3.6 MVar compared to the PI control scheme. For the MPC scheme, it can reach 2.222 MVar and 4.026 MVar respectively. The simulation results indicate the proposed control scheme shows superior performance during the LVRT and the subsequent HVRT events, which can fast reduce voltage deviations while achieving more effective and faster reactive power support from the WF by optimal droop control.

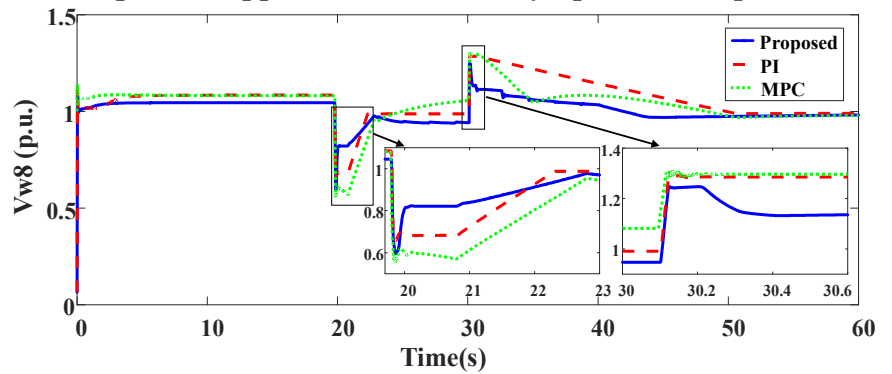

(a)

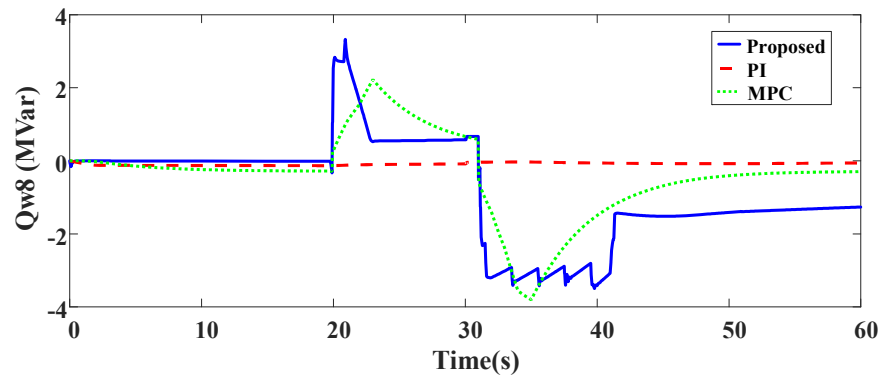

(b)

Fig. 18. Control performances of WT8 under subsequent HVRT and LVRT events, (a) Voltage profile, (b) Reactive power output.

Fig. 19 shows comparative control performances of the WT considering the variation of wind speed (CVWS) and without considering the variation of wind speed (WCVWS). It is shown that the three control schemes have a better overvoltage suppression effect at lower speed because the DFIG has a larger reactive power capacity and thus can absorb more reactive power.

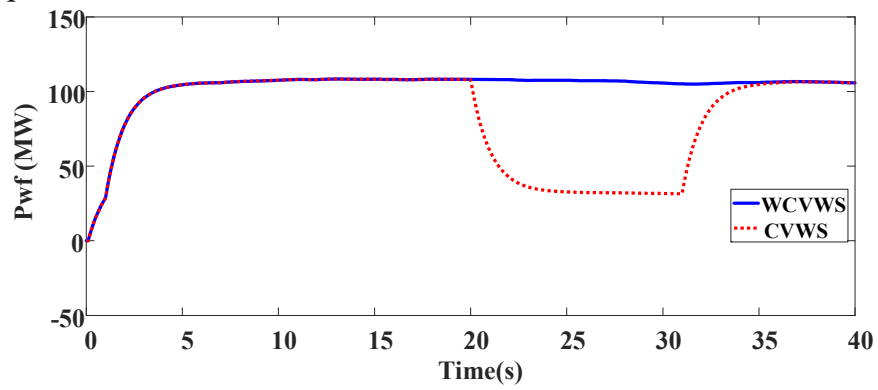

(a)

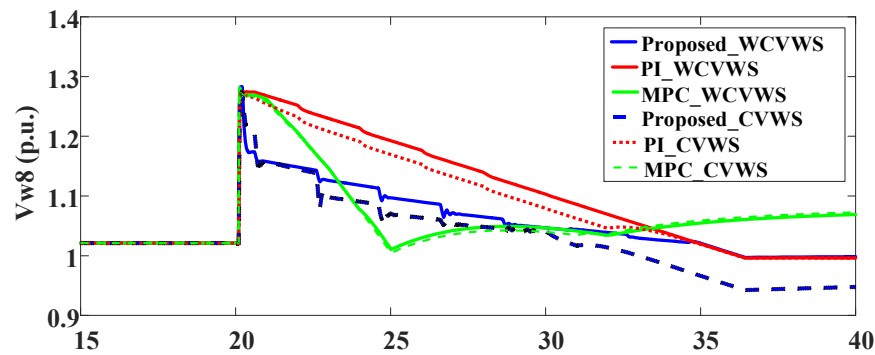

(b)

Fig. 19. Control performances considering the variation of wind speed, (a) Active power output of WF, (b) Voltage profile of WT8.

\section{Control Performance Under Asymmetrical faults}

In order to evaluate the effectiveness of the proposed control scheme under asymmetrical faults, a detailed nonlinear DFIG model is established. The comparison study is conducted between the two cases: 1) Case 1 is a symmetrical grid voltage swell of 1.25 p.u.; 2) Case 2 is an asymmetrical grid voltage swell of 1.25 p.u.. The simulation results are shown in Figs. 2022.

Fig. 20 shows the voltage profile of the WT8 terminal bus in Cases 1 and 2. After the voltage swell event occurs, the WT8 terminal voltages under the two cases all have a sudden increase. Moreover, the voltage fluctuation in Case 1 is larger than that in Case 2. Fig 21 shows the reactive current of WT8. From $20.1 \mathrm{~s}$ to $30.1 \mathrm{~s}$, the reactive current increases gradually with the reactive power reference increasing. As can be seen from Fig 22, the control mode switches from the normal operation to the HVRT control mode at $\mathrm{t}=20.1 \mathrm{~s}$. At $\mathrm{t}=20.6 \mathrm{~s}$, the control mode switches to the post-event recovery control mode. The controllers switch to the normal mode at $\mathrm{t}=30.1 \mathrm{~s}$ and keep stable in the remaining period.

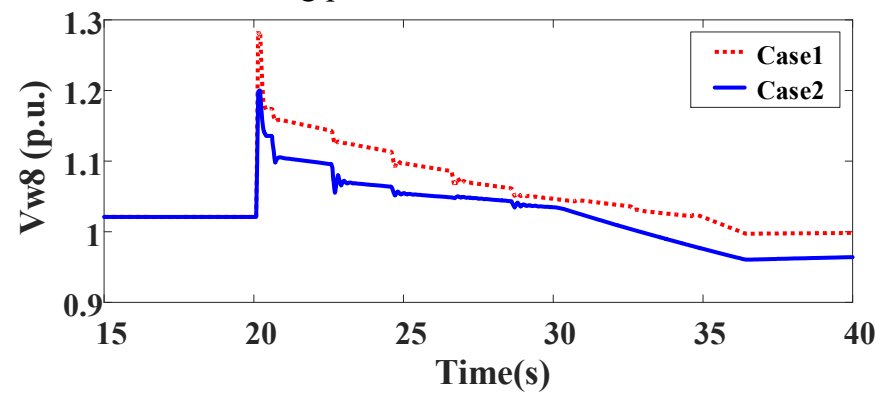

Fig. 20. Voltage profile of WT8 terminal bus in Cases 1 and 2. 


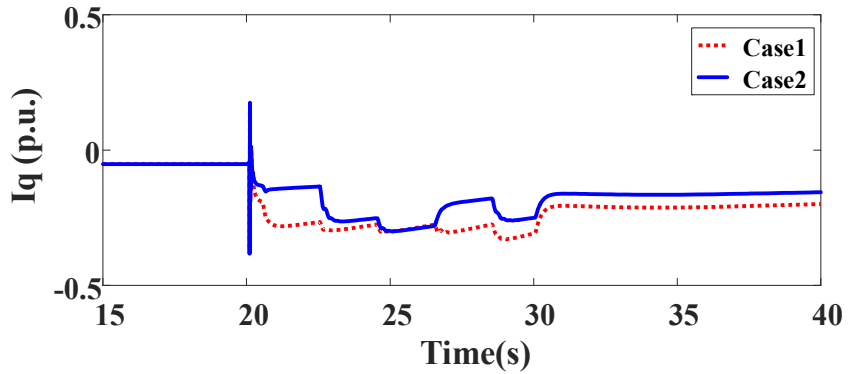

Fig. 21. Reactive current of WT8.

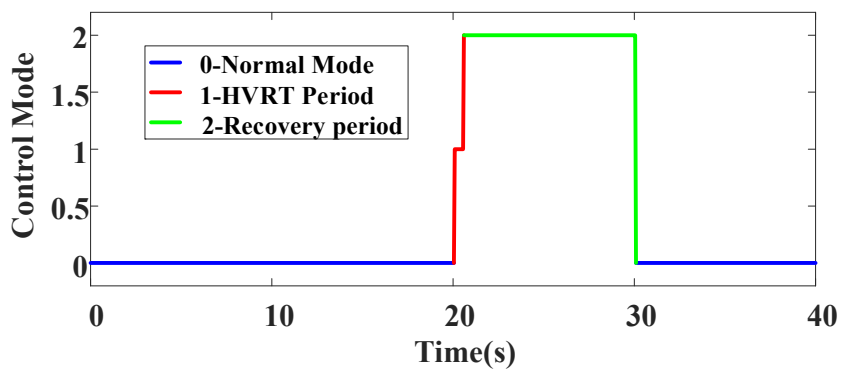

Fig. 22. Control mode switching.

\section{CONCLUSION}

In this paper, an optimal coordination of droop control and adaptive model predictive control scheme is proposed for enhancing the HVRT and post-event recovery capability of large-scale WFs to handle the overvoltage of the WT terminal. The optimal droop control during the HVRT can achieve the fast WT terminal voltage reduction by tracking the optimal droop coefficients. The MPC algorithm is coordinated with the local droop control to optimize the reactive power output of each WT inside the WF and realize the rapid post-event recovery. The proposed control scheme can achieve a better global optimal performance while reducing the communication and calculation burden of the WF central controller. The simulations show that the proposed control scheme achieves a more effective and faster terminal over-voltage mitigation of WTs during the HVRT and the post-event recovery period. The terminal voltage oscillation is reduced, and the time during HVRT and post-event recovery is much shorter compared with the PI and MPC schemes.

\section{REFERENCES}

[1] W. Li, M. Zhu, P. Chao, X. Liang, and D. Xu, "Enhanced FRT and postfault recovery control for MMC-HVDC connected offshore wind farms," IEEE Trans. Power Syst., vol. 35, no. 2, pp. 1606-1617, Mar. 2020.

[2] D. Xu, Q. Wu, B. Zhou, C. Li, L. Bai, and S. Huang, "Distributed multienergy operation of coupled electricity, heating and natural gas networks," IEEE Trans. Sustain. Energy, vol. 11, no. 4, pp. 2457-2469, Oct. 2020.

[3] S. Huang, Q. Wu, Y. Guo, X. Chen, B. Zhou, and C. Li, "Distributed Voltage Control based ADMM for Large-Scale Wind Farm Cluster connected to VSC-HVDC," IEEE Trans. Sustain. Energy, vol. 11, no. 2, pp. 584-594, Apr. 2020.

[4] P. Kundur, "Power system stability and control," 1994, McGraw-Hill, Inc., ISBN-13: 978-0-07-035958-1.

[5] M. Shi, X Chen, J. Zhou, Y. Chen, J. Wen, and H. He, "Advanced secondary voltage recovery control for multiple HESSs in a droopcontrolled DC microgrid," IEEE Trans. Smart Grid, vol. 10, no. 4, pp. 3828-3839, Jul. 2019.

[6] P. Dash, R. Patnaik, and S. Mishra, "Adaptive fractional integral terminal sliding mode power control of UPFC in DFIG wind farm penetrated multimachine power system," Protect. Control Mod. Power Syst., vol. 3, no. 8, pp. 1-14, Mar. 2018.
[7] M. Raza, C. Collados, and O. Bellmunt, "Reactive power management in an offshore AC network having multiple voltage source converters," Appl Energy, vol. 265, pp. 793-803, Nov. 2017.

[8] J. Ruan, Z. Lu, Y. Qiao, and Y. Min, "Analysis on applicability problems of the aggregation-based representation of wind farms considering DFIGs' LVRT behaviors," IEEE Trans. Power Syst., vol. 31, no. 6, pp. 4953-4965, Nov. 2016.

[9] Q. Zhou, Y. Cheng, and X. Bian, "Analysis of restrike overvoltage of circuit breakers in offshore wind farms," IEEE Trans. Appl. Supercond., vol. 26, no. 7, pp. 1-5, Nov. 2016.

[10] H. Zhao, Q. Wu, J. Wang, Z. Liu, M. Shahidehpour, and Y. Xue, "Combined active and reactive power control of wind farms based on model predictive control," IEEE Trans. Sustain. Energy, vol. 32, no. 3, pp. 1177-1187, Sep. 2017.

[11]M. Mansour, M. Mohammad, and S. Islam, "Low and high voltage ridethrough of DFIG wind turbines using hybrid current controlled converters," Electr. Power Syst. Res., vol. 81, no. 7, pp. 1456-1465, Jul. 2011.

[12]C. Feltes, S. Engelhardt, J. Kretschmann, J. Fortmann, F. Koch, and I. Erlich, "High voltage ride through of DFIG based wind turbines," in Proc. IEEE Power Energy Soc. Gen. Meet., Pittsburgh, PA, USA, pp.1-8, Aug. 2008

[13]Z. Xie, X. Zhang, X. Zhang, S. Yang, and L. Wang, "Improved ridethrough control of DFIG during grid voltage swell," IEEE Trans. Ind. Electron., vol. 62, no. 6, pp. 3584-3594, Jun. 2015.

[14] J. Yang, J. Fletcher, and J. Reilly, "A series dynamic resistor based converter protection scheme for doubly fed induction generator during various fault conditions," IEEE Trans. Energy Covers., vol. 25, no. 2, pp. 422-432, Feb. 2010.

[15]L. Sun, and Y. Wang, "Study on transient whole-process and control strategy of Stator flux in doubly-fed induction generator with asymmetric voltage swell," High Voltage Engine., vol. 45, no. 7, pp. 2160-2166, Jul. 2019.

[16]B. Karthikeya and R. Schutt, "Overview of wind park control strategies," IEEE Trans. Sustain. Energy, vol. 5, no. 2, pp. 416-422, Apr. 2014.

[17]H. Zhao, Q. Wu, Q. Guo, H. Sun, S. Huang, and Y. Xue, "Coordinated voltage control of a wind farm based on model predictive control," IEEE Trans. Sustain. Energy, vol. 7, no. 4, pp. 1440-1451, Oct. 2016.

[18] Y. Guo, H. Gao, Q. Wu, H. Zhao, J, Ostergaard, and M. Shahidehpour, "Enhanced voltage control of VSC-HVDC connected offshore wind farms based on model predictive control," IEEE Trans. Sustain. Energy, vol. 9, no. 1, pp. 474-487, Jan. 2018.

[19] Q. Zhou, M. Shahidehpour, A. Paaso, S. Bahramirad, A. Abdulwhab, and A. M. Abusorrah, "Distributed control and communication strategies in networked microgrids," IEEE Commun. Surveys Tuts., early access, 2020, doi: 10.1109/COMST.2020.3023963.

[20] Q. Zhou, M. Shahidehpour, Z. Li, L. Che, A. Abdulwhab, and A. M. Abusorrah, "Compartmentalization strategy for the optimal economic operation of a hybrid ac/dc microgrid," IEEE Trans. Power Syst., vol. 35, no. 2, pp. 1294-1304, Mar. 2020

[21] Y. Guo, H. Gao, H. Xing, Q. Wu, and Z. Lin, "Decentralized coordinated voltage control for VSC-HVDC connected wind farms based on ADMM," IEEE Trans. Sustain. Energy, vol. 10, no. 2, pp. 800-810, Apr. 2018.

[22] S. Huang, Q. Wu, J. Zhao, and W. Liao, "Distributed optimal voltage control for VSC-HVDC connected large-scale wind farm cluster based on analytical target cascading method," IEEE Trans. Sustain. Energy, vol. 11, no. 4, pp. 2152-2161, Oct. 2020.

[23] GB / T 36995-2018. Wind turbines-Test procedure of voltage fault ride through capability. China, Dec. 2018.

[24] E.O.N. Netz. Grid code-high and extra high voltage. E. ON Netz GmbH, Bayreuth, Apr.2006.

[25]J. Mart'ınez, P. C. Kjær, and R. Teodorescu, "DFIG turbine representation for small signal voltage control studies," in proc. 12th Int. Conf. on Optimization of Electri. and Elect. Equip., IEEE, pp. 31-40. May 2010.

[26] X. Liu, C. Li, M. Shahidehpour, Y. Gao, and B. Zhou, "Fault current hierarchical limitation strategy for fault ride-through scheme of microgrid," IEEE Trans. Smart Grid, vol. 10, no. 6, pp. 6566-6579, Mar. 2019.

[27]K. Christakou, J. LeBoudec, M. Paolone, and D. Tomozei, "Efficient computation of sensitivity coefficients of node voltages and line currents in unbalanced radial electrical distribution networks," IEEE Trans. Smart Grid, vol. 4, no. 2, pp. 741-750, Jun. 2013.

[28]J. Grunnet, M. Soltani, T. Knudsen, M. Kragelund, and T. Bak, "Aeolus toolbox for dynamic wind farm model, simulation and control," in Proc. Eur. Wind Energy Conf., 2010, pp. 1-6.

[29]Y. Guo, H. Gao, and Q. Wu, "Distributed cooperative voltage control of wind farms based on consen-sus protocol," Int. J. Electr. Power Energy Syst., vol. 104, pp. 593-602, Jan. 2019. 